\title{
Belgeo
}

Revue belge de géographie

\section{La production d'une représentation touristique. Une objectivation des guides Lonely Planet sur le Groenland}

The production of a tourist representation. An objectification of the Lonely

Planet guides on Greenland

\section{Antoine Delmas}

\section{CpenEdition}

\section{Journals}

Édition électronique

URL : http://journals.openedition.org/belgeo/7207

DOI : 10.4000/belgeo.7207

ISSN : 2294-9135

Éditeur :

National Committee of Geography of Belgium, Société Royale Belge de Géographie

Référence électronique

Antoine Delmas, "La production d'une représentation touristique. Une objectivation des guides Lonely Planet sur le Groenland », Belgeo [En ligne], 3 | 2012, mis en ligne le 18 mars 2013, consulté le 30 avril 2019. URL : http://journals.openedition.org/belgeo/7207; DOI : 10.4000/belgeo.7207

Ce document a été généré automatiquement le 30 avril 2019.

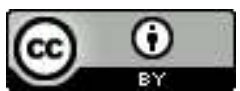

Belgeo est mis à disposition selon les termes de la licence Creative Commons Attribution 4.0 International. 


\title{
La production d'une représentation touristique. Une objectivation des guides Lonely Planet sur le Groenland
}

\author{
The production of a tourist representation. An objectification of the Lonely \\ Planet guides on Greenland
}

Antoine Delmas

\section{Introduction}

1 Le Groenland, littéralement la "terre verte", accueille annuellement un peu plus 70000 touristes. La venue de ces visiteurs se fait principalement au cours de la saison estivale qui s'étend de juin à août. S'ajoute à cet afflux épisodique, la venue providentielle de touristes adeptes du traîneau à chiens au début du printemps. À l'année, la clientèle touristique de ce territoire autonome danois se distingue selon trois catégories. La première se différencie autour des croisiéristes dont nous pouvons isoler trois soustypes: les navires transatlantiques de plusieurs milliers de passagers pour lesquels le Groenland est l'une des étapes du séjour ; les bateaux de plusieurs centaines de touristes, et les croisières d'expédition à bord desquelles une centaine de passagers tout au plus s'adonnent à une découverte méticuleuse de l'île. La seconde catégorie se forme autour des circuits individuels terrestres, semi-organisés ou non. Les touristes rayonnent depuis un site touristique principal dans un périmètre restreint afin d'effectuer des excursions de courte durée, d'une à quelques journées. Cette clientèle est aisée en raison des prestations le plus souvent personnalisées: activités guidées, hôtel de standing, restaurant, etc.

2 Ces deux premières catégories se distinguent de la troisième, les backpackers ou routards qui incarnent la clientèle historique du Groenland depuis les débuts de la mise en 
tourisme dans les années 1970. Ils voyagent parfois seuls, mais ont aussi recours à des expéditions guidées dites "d'aventure" en raison des difficultés à planifier avec précision l'intégralité d'un voyage sans bénéficier de contacts locaux.

3 Animés par un désir de distinction, les backpackers recherchent des formes touristiques disjonctives aux pratiques dominantes - de masse, de luxe, de croisière, etc. - via des activités alternatives (Maoz, 2007). La conjonction entre deux phénomènes, d'un côté une envie de distinction encouragée par des facteurs sociaux - recherche de sa propre identité, ressourcement, désir de se couper du monde (Noy, 2004 ; Maoz, 2007 ; Pearce et Foster, 2007) - et de l'autre l'apparition d'ouvrages destinés aux backpackers, a participé à une démocratisation rapide de ce mode de voyager après son émergence dans les années 1960. Distillant conseils, bons plans, lieux à visiter, le Guide du Routard, dans quelques pays francophones, et surtout les ouvrages édités par Lonely Planet à l'échelle internationale incarnent les guides de référence pour les touristes à petit budget ou les backpackers. Le premier ouvrage de Lonely Planet de 1973 retraçait le périple du couple fondateur de la maison d'édition Tony et Maureen Wheeler, de l'Angleterre à l'Australie. Aujourd'hui la philosophie de la société australienne a profondément évolué. Les premiers guides publiés en anglais ont cédé leur place à une collection de 500 ouvrages couvrant 195 pays et traduits en 8 langues. Aucune destination ne semble échapper à la couverture méticuleuse établie par l'éditeur ; si bien que des destinations inattendues, en proie à une insécurité prégnante, telles que l'Afghanistan, la Somalie ou encore la Sierra Leone, disposent de quelques pages dans des guides transnationaux. Des destinations à la marge des flux touristiques mondiaux sont, elles aussi, l'objet de guides tel que le Groenland. Sujet d'un guide pour la première fois en 1991, le fait touristique y a été abordé ensuite à cinq reprises : 1994, 1997, 1999, 2001 et 2005. Deux éditions différentes ont été publiées conjointement. La première, rééditée quatre fois, se consacrait en premier lieu à l'Islande ; le Groenland y était abordé en tant que destination secondaire. Parallèlement, en 1999, Lonely Planet a publié un autre guide consacré à l'ensemble des hautes latitudes septentrionales : Canada, États-Unis, Russie, Finlande, Suède, Norvège et Groenland. Toutefois, cet ouvrage aborde le Groenland succinctement (25 pages) et a été, pour cette raison, écarté de nombres d'analyses menées dans cet article. La deuxième édition, datée de 2005, s'est exclusivement focalisée sur le Groenland. C'est, à ce jour, le premier et dernier guide dans lequel l'île est au cœur de l'ouvrage. Nous le comprenons, la place du Groenland en une quinzaine d'années a été modifiée. Initialement traitée comme une destination secondaire de 1991 à 2001, en 2005 l'île bénéficie d'un guide qui lui est en grande partie dédié. Une des caractéristiques de ces ouvrages est la permanence des informations d'une édition à l'autre, voire même la réutilisation intégrale de portions de textes précédemment publiés. Ce constat rejoint le mode de rédaction minimaliste des ouvrages, qui procèdent à une synthèse des informations disponibles et ne se livrent pas une véritable enquête de terrain (Bonin, 2001 ; Ollivier, 2012).

Publiés uniquement en langue anglaise, ces guides s'affirment comme la référence internationale des ouvrages sur l'île. La dernière édition est épuisée des circuits de distribution traditionnels; elle se vend d'occasion à des tarifs qui dépassent la centaine de dollars, soit cinq fois le prix initial. Lonely Planet n'est pas le seul éditeur à s'intéresser au Groenland ; d'autres initiatives ont vu le jour à l'image des deux éditions du guide produit par l'agence de voyage française Grand Nord Grand Large ou bien encore l'ouvrage anglophone dédié exclusivement à un itinéraire de randonnée qui sillonne l'ouest du 
Groenland. Cependant, ces guides n'ont pas acquis la notoriété de ceux édités par Lonely Planet et se retreignent à une sphère de diffusion confidentielle.

Outils de prises de décision du voyageur, ne pourrions-nous pas étudier rigoureusement les guides comme un matériel scientifique à part entière ? Détourner le sens commun de lecture nécessite de considérer les informations du guide, non pas comme un contenu subjectif mais comme une représentation objective du territoire (Bonin, 2001). Ce parti pris scientifique peut être décrié en raison de l'apparente partialité de ces ouvrages qui orientent le regard et l'attention du touriste sur certains lieux et offrent parfois des visions stéréotypées. Malgré ces légitimes critiques, les guides photographient, au moment de leur conception, la réalité locale et s'affirment alors comme les témoins directs de la mise en tourisme d'un territoire. Quand bien même cet argument ne serait pas suffisant à convaincre les esprits peu enclins à une telle démarche, l'éventuelle subjectivité que l'on peut reprocher aux guides peut être au cœur de l'analyse en mettant au jour un double mécanisme. Les propos de ces ouvrages sont façonnés par le lieu social des rédacteurs et par la représentation qu'ils se créent du territoire considéré (Baider, 2003 ; Bonin, 2001; Guillet, 2003; Ollivier, 2012) et ces derniers modèlent leurs discours au regard de la clientèle qu'ils visent, afin de mieux la séduire ou de lui présenter la réalité qu'elle est susceptible d'attendre (Bonin 2001 ; Chevalier, 1975).

Ainsi, l'analyse des guides sera un moyen d'étudier le décalage présent entre la représentation et la réalité territoriale. Identifier les thématiques valorisées ou au contraire dénommer les sujets minorés, revient à s'interroger sur la représentation publique (Sperber, 1989), c'est-à-dire le medium qui se crée entre les auteurs et les lecteurs. Son identification se fera via une analyse de contenu qui mêlera l'approche quantitative se concentrant sur les fréquences d'apparition des thématiques considérées et l'approche qualitative dans laquelle c'est la dimension nouvelle, la valeur d'un thème, la relation aux autres qui suscitent le plus d'intérêt (Grawitz, 1989). 
Figure 1. Localisation des villes et régions citées dans le contenu de l'article (Conception et Réalisation : AD).

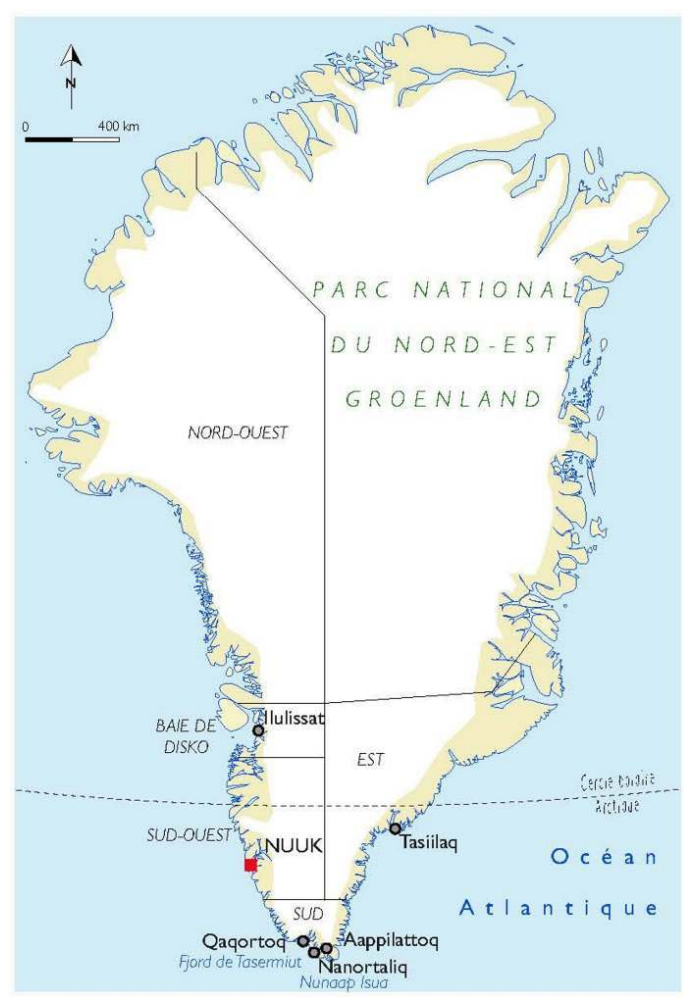

\section{Un besoin irrépressible de distinction}

7 Afin de maximiser les chances de réussite commerciale d'un guide, les auteurs se doivent de satisfaire les attentes des lecteurs, quitte à déformer partiellement la réalité. Ainsi les premiers ouvrages de Lonely Planet consacrés au Groenland adoptent une posture critique vis à vis du tourisme. L'ambiguïté de cette attitude peut de prime abord surprendre, mais elle rentre pourtant en résonnance avec les attentes singulières des backpackers. Ces derniers cherchent à la fois à s'autonomiser des formes touristiques majoritaires qui se contenteraient de l'observation passive d'un environnement sans en comprendre les enjeux sociaux, économiques et environnementaux, et à faire émerger une identité propre au backpacking (Leroux, 2007). Dans les faits, la critique mise en place par les auteurs associe deux dimensions : une distinction géographique - désir de fuir les lieux touristiques - et une distinction sociale - désapprouver les pratiques des autres touristes. Au fil des éditions, les critiques ont été croissantes pour atteindre leur paroxysme en 2005. Dans cet ouvrage, les auteurs y sont les plus virulents et manifestent expressément leurs regrets face au développement touristique de l'île.

Les principaux sites en proie au "tourisme ordinaire" auraient perdu de leur charme et de leur authenticité. Ils ne seraient d'ailleurs plus d'un intérêt majeur. Ainsi, dans la phase introductive du chapitre consacré à Ilulissat, principale ville touristique du Groenland, les auteurs marquent leur préférence pour une toute autre région située à l'écart des circuits touristiques :

However scruffy and unkempt, Ilulissat (The Icebergs) is the Arctic you came to see

- cold, mirror-like seas crowded with icebergs and floes, an often unrelenting grey 
sky and disorderly spirit noticeably missing from the tidier towns farter south.

Éditions de 1991, 1994, 1997, 1999 et 2001

9 La manifestation la plus concrète de la valorisation du sud est la longueur du chapitre qui lui est consacré: un quart de l'ouvrage, alors qu'il existe cinq grandes régions touristiques dans les guides : sud, sud-ouest, baie de Disko, nord-ouest et est. Qaqortoq, plus importante ville de la région sud du Groenland, quatrième entité dans la hiérarchie urbaine, est décrite avec éloge :

Many visitors find it the cleanest and tidiest of all Greenlandic towns, and in midsummer it explodes with wildflowers.

Éditions de 1994, 1997, 1999 et 2001

Tout comme la ville de Nanortalik, située à quelques encablures de Qaqortoq :

If you only have a short holiday in Greenland, you may want to consider spending it in Nanortalik, which has some of Greenland's friendliest people and most spectacular surroundings.

Éditions de 1994, 1997, 2001

11 Pour motiver cette politique éditoriale, les auteurs soulignent la singularité des paysages mais, en filigrane, c'est surtout l'absence de touristes qui les amène à focaliser leur attention sur cette portion de l'île :

Blissfully scenic South Greenland is far more than a touristic also-ran to the big ice of Disko Bay. The bergs may be smallest, but the mountains are much more impressive, coming to an astonishing scenic climax.

Édition de 2005

12 Le petit village d'Aapilatoq - dont les descriptions lyriques traduisent l'intérêt du site “ superbly photogenic (...) astounding panorama (...) Greenland's most beautiful inland waterways" - semble, pour les auteurs, être doté d'un atout indéniable qui induit son caractère originel : l'absence de tourisme.

Unbelievably untouched by tourism, economy revolves around seal hunting.

Édition de 2005

13 À ce désir de distinction géographique s'ajoute une critique sociale des touristes et de leur comportement. Les guides concentrent leurs reproches sur les excursionnistes ou day trippers. Depuis trois décennies, ces visiteurs profitent de la proximité géographique de l'Islande pour se rendre quelques heures voire quelques jours dans la région est du Groenland. Selon les auteurs, les excursionnistes débarquent, photographient, achètent un souvenir et repartent sans comprendre la nature même de la destination, sans véritablement la découvrir. Ainsi, selon eux, cette forme de tourisme entraîne un voyeurisme exacerbé :

Taking photos is no problem but, on a day tour, it may involve some jostling!

Édition de 1994,1997 et 2005

14 Les guides encouragent à se distinguer de ces derniers en restant plusieurs jours sur la destination dans le but de mieux comprendre le quotidien des habitants, de mieux s'en imprégner :

To experience real life here, stay overnight and explore when the day trippers have

left.

Édition de 2005

Indéniablement pour les auteurs, la longueur du séjour devient un moyen de distinction entre excursionnistes et autres voyageurs. Mais il est faux de concevoir la durée du séjour comme une condition sine qua non permettant d'accéder à une meilleure compréhension de la vie locale. Ce débat renvoie à l'opposition traditionnelle qui est faite entre quantité 
et qualité des plaisirs, pourtant tranchée depuis longtemps par les deux pères fondateurs de l'utilitarisme Jérémy Bentham et John Stuart Mill (préface de Francisco Vergara de La Nature, Mill, 2003). Ces deux penseurs insistent sur le fait que c'est la qualité et non la quantité d'un plaisir qui permettra d'en mesurer la valeur. La durée n'influe pas sur la qualité des séjours mais bel et bien sur la "quantité" de temps passé à voyager. Elle ne doit pas être considérée comme une valeur intrinsèque mais comme une donnée supplémentaire qui ne préjuge en rien d'une meilleure connaissance des lieux.

Les auteurs, sous couvert de remettre en cause la pratique des day trippers, ne sont pas exempts de critiques envers leurs propres lecteurs. Dans un encadré, après avoir dénoncé le comportement des excursionnistes, ils prodiguent à leur lectorat des conseils de bonne conduite : ne pas développer une empathie démesurée :

In the past well-meaning visitors have given sweets, money or pens to Greenlandic children, but this patronishing behaviour upsets parents and demeans the children by inspiring them to ask subsequent visitors for more of the same. Please resist the temptation to play Santa Claus, and let traditional life survive as the locals see fit.

Édition de 1999, 2001 et 2005

Les critiques lancées à l'égard de l'activité touristique permettent d'identifier l'un des postulats des ouvrages : marquer la distance avec les autres visiteurs. Ces considérations sont un préalable nécessaire avant de livrer une étude détaillée du contenu des guides.

\section{Une immersion dans la wilderness}

Façonnés par la rudesse du climat et par l'écoulement des glaciers, les paysages groenlandais se révèlent d'une diversité insoupçonnée loin de la représentation commune qui les réduit aux seuls icebergs. Les montagnes et les paysages du froid restent omniprésents, mais leur diversité est tout aussi remarquable. Le sud s'apparente aux fjords norvégiens. Le centre ouest se distingue par la présence de moyennes montagnes côtières aux flancs parsemés de lacs et d'espaces marécageux tandis que les eaux marines sont parcourues d'icebergs. Le grand quart sud-est est le territoire du gigantisme, des reliefs marqués et des plus imposants fjords du globe. Enfin, le nord de l'île se caractérise par la minéralité de ses paysages, dont la partie est constitue le plus grand parc national du monde. Les auteurs se sont saisis de cette diversité pour la mettre en exergue, mais leur attention reste focalisée sur la partie sud de l'île. Comme dans tout guide, les descriptions sont élogieuses, parfois même lyriques, et participent à une exaltation des sentiments du lecteur. Les expressions employées esthétisent les paysages et soulignent leur hostilité afin de faire percevoir au "lecteur-visiteur", conformément à ses attentes, une sensation de vulnérabilité dans un environnement sauvage :

Two of the world's highest and most impressive granit faces. Édition de 1997 et 2001

The dramatic Tasilaq-arealandscape is a natural attraction (...) Édition de 1997, 1999

et 2001

(...) a basin backed by cliff walls and split by gash-like canyons and low, ruged hills.

Édition 1991, 1994, 1997, 1999, 2001 et 2005

19 La sémantique utilisée renvoie à la notion anglo-saxonne de wilderness qui ne se réfère ni à la "nature sauvage", ni à la "sauvagerie", ni à la "naturalité", mais qualifie le "sauvage c'est-à-dire cette nature laissée à son propre sort et des individus jugés primitifs" (Arnould et Glon, 2006). Le contexte d'émergence socio-historique de la notion, l'arrivée de colons européens découvrant l'Amérique du Nord, permet de comprendre que la wilderness est l'apanage d'une position dominante (Arnould et Glon, 2006). Ainsi, elle 
n'existe que dans l'œil de l'étranger qui découvre une réalité qu'il connaît mal et dont les capitaux sociaux et culturels ne lui permettent pas de se saisir. Pour les Groenlandais, leur île n'est pas si hostile et ce en raison du mode de vie qu'ils pratiquent en harmonie avec les contraintes imposées par les facteurs naturels. La vision des auteurs est donc empreinte de considérations occidentales qui influent sur le discours des guides.

Dans les années 1990 et au début des années 2000, les paysages du Groenland restaient encore méconnus du grand public. Ainsi, pour que les touristes se forgent une représentation des panoramas qui les attendent, les auteurs ont établi de nombreuses analogies : le cap le plus méridional de lîle (Nunaap Isua) était comparé au cap Horn, les falaises du sud de l'île évoquaient la mythique et intimidante face d'El Capitan dans le parc national du Yosemite ou encore les montagnes situées dans le massif du Karakoram.

The immense granite peaks and spires of the Nanortalik and Nunaap Isua areas are destinated to be compared with those of Chilean Patagonia or Yosemite. Even comparisons fall short, however; this is a most spectacular place.

Éditions de 1991, 1994 et 1997

21 Le fait que les auteurs soulignent le manque de pertinence des comparaisons, rehausse l'importance de la wilderness groenlandaise et la singularise des grands emblèmes paysagers cités. Les analogies, moins récurrentes en 2005, marquent la fin du "déficit d'images" que connaissait le Groenland jusqu'alors. Préoccupation majeure du 21e siècle, le changement climatique a attiré l'attention des médias sur des sites particulièrement menacés par cette nouvelle donne mondiale. Le Groenland en incarne l'un des emblèmes et c'est, entre autres, pour cette raison, qu'une année auparavant, le fjord glacé d'Ilulissat a été classé au patrimoine mondial de l'UNESCO.

Pour les auteurs, se confronter à la wilderness ne peut se faire que par le biais de pratiques touristiques d'aventure. Les sommets du fjord de Tasermiut, la région du Nunaap Isua ou bien encore les montagnes vierges de l'est, sont des hauts-lieux de l'alpinisme ; la région de Nanortalik devient "La Mecque" pour les montagnards, grimpeurs et autres kayakistes. Les falaises de cette région sont d'ailleurs le spot à la mode pour la pratique du base-jump. En plus de ces pratiques engagées, les auteurs évoquent, à de multiples reprises, les dangers encourus au Groenland. Lors des descriptions d'itinéraires de randonnée, activité par ailleurs plébiscitée dans les ouvrages, les auteurs multiplient les conseils et avertissent leurs lecteurs des potentiels dangers qu'ils encourent: variabilité météorologique, difficultés d'orientation, etc. Les guides rappellent même que si les touristes ont recours à des équipes de sauvetage, dans la plupart des cas le défraiement des coûts induits sera à leur charge. Les dangers que représentent les activités extérieures sont indéniables, mais la présentation emphatique qui en est faite renforce le sentiment de vulnérabilité du lecteur. L'émergence d'un imaginaire touristique, où l'incertitude et le danger sont au cœur du voyage, est conforme à la quête des backpackers qui cherchent à diversifier leurs expériences par le risque (Elsrud, 2001).

La définition de wilderness avancée par Paul Arnould et Éric Glon en 2006 fait état de l'importance de l'environnement physique, mais aussi de la place prépondérante prise par les "individus jugés primitifs". En raison de l'évolution de la pensée scientifique, notamment grâce aux travaux de Claude Lévi-Strauss (1961), le terme de primitifs ne peut plus être utilisé de nos jours et, s'il l'était, cela traduirait une posture d'esprit passéiste voire même révisionniste. Toutefois, en raison de la singularité de la culture groenlandaise, sa découverte permet de découvrir une nouvelle facette de la destination. 


\section{Une valorisation de la culture}

24 À tort, nous dénommons les habitants du Groenland Inuit, littéralement les hommes. Cette appellation générique confond dans un même ensemble les peuples autochtones originaires du nord du Canada, de la Sibérie et du Groenland. Précisément, les habitants de l'île se dénomment Kalaallit. La culture de ces derniers dispose de nombreux traits communs avec celles des Inuit ${ }^{1}$, à l'image des mythes fondateurs, mais présente aussi des éléments de différenciations et notamment la langue, le kalaallisut. Les spécificités et les particularismes propres à la culture groenlandaise sont autant d'éléments d'autonomisation que de points forts pour affirmer une identité commune aux latitudes septentrionales.

La rencontre avec la culture groenlandaise est quantitativement la seconde thématique abordée dans les guides après la découverte des paysages. Sa place a été grandissante au fil des éditions, ce qui traduit un enrichissement de l'offre touristique du Groenland pour satisfaire la venue croissante de touristes entre 1991, date de la première édition et $2005^{2}$. Ainsi, sans la simplifier ni la résumer, l'avant-propos, consacré à la description générale de l'île, dresse un aperçu exhaustif et représentatif de la réalité culturelle locale. Cet éclaircissement répond pleinement au désir des lecteurs-cibles, les backpackers, qui aspirent à mieux comprendre la destination afin d'en saisir toute sa complexité. Cette recherche serait d'ailleurs l'une des caractéristiques de cette clientèle (Maoz, 2007). Sur le terrain, les artisans ont transformé leurs produits - sculptures, peaux tannées, vêtements traditionnels -, cadre de la vie locale, en objets de souvenirs valorisés économiquement. Mentionnés à seulement cinq reprises dans le guide de 1991, les auteurs ont, en 2005, identifié vingt-sept lieux d'achats de souvenirs "authentiques".

Au fil des éditions, les auteurs ont noté l'émergence de nouvelles contingences pour rentrer dans l'intimité de la vie culturelle des Groenlandais : chant, défilé en costume, partage d'un repas, pêche sur glace, etc. Ces moments forts de la vie de la communauté sont devenus des moments à partager, à présenter aux touristes, afin de les immerger dans la culture locale. Cette mise en avant répond au désir d'authenticité et encore plus à la "quête originelle" à laquelle se livre les touristes. Décrit par Véronique Antomarchi, ce processus se réfère à la volonté de retrouver les origines primitives des sociétés occidentales (Créquy, 2010). Pour les backpackers, ces voyageurs à la recherche de leur propre identité, cette quête est une posture indissociable de la pratique touristique (Maoz, 2007 ; Noy, 2004). Néanmoins, Alain Grenier (2008) rappelle que l'ensemble des touristes recherche l'authenticité et que ce n'est en aucun cas la particularité de certains. Cette quête est donc un désir partagé. Chaque touriste, au regard de ses exigences, en détermine le degré escompté: quelques-uns se contenteront de l'achat d'un souvenir, d'autres souhaiteront partager un repas avec les habitants, tandis que les derniers s'immergeront pleinement dans l'environnement visité. Le doute émis par Alain Grenier (2008) est réaffirmé par Larsen et alii (2011) qui minimisent, grâce à une vaste étude quantitative, les singularités des backpackers et soulignent la surinterprétation des éléments de distinction. L'étude des brochures touristiques proposées dans les agences de voyage et les enquêtes de terrain menées au Groenland durant l'été $2011^{3}$ aboutissent à un constat semblable. Les pratiques des backpackers et des autres touristes jouent sur un registre semblable articulant wilderness et culture. Ainsi, le désir de distinction des backpackers, entretenu par les guides, n'est plus une réalité mais se rapproche d'un idéal à 
atteindre; l'offre touristique destinée initialement à ces visiteurs historiques s'est progressivement déployée à l'ensemble de la clientèle, inhibant le désir de singularisation et fragilisant "l'entre-soi" voulu par les backpackers.

\section{Conclusion}

Bien plus qu'une invitation aux voyages, l'objectivation des guides touristiques comme matériel scientifique a permis de dresser le portrait touristique du Groenland déformé au prisme des auteurs de Lonely Planet. Une analyse géographique, inspirée par une pluralité de méthodes issues d'autres sciences sociales, possède toutes les clefs pour décrypter scientifiquement le contenu de ces ouvrages. La récurrence des mentions sur la beauté des paysages ne laisse aucun doute possible, le Groenland est une destination où les attraits naturels prédominent. Toutefois, les guides témoignent des orientations stratégiques prises par les acteurs locaux pour adjoindre à la richesse naturelle, la possibilité de découvrir une culture méconnue. Cette mise en valeur révèle deux phénomènes concomitants : tout d'abord la prise de conscience, de la part des habitants, de leur spécificité et de la volonté des auteurs des guides d'adjoindre au Groenland une image de destination culturelle. Le désir de distinction et la nécessité de créer une identité collective propre aux backpackers apparaissent comme les fils conducteurs des ouvrages. Malgré les doutes émis par certains chercheurs quant à la pertinence de cette identité, la création de l'“entre-soi" témoigne de "l'extériorisation de l'intériorité" décrite par Bourdieu : affirmer sa différence pour mieux se l'approprier.

Malgré une augmentation de $18 \%$ de la fréquentation touristique entre 2001 et 2011, l'isolement géographique reste une contrainte majeure pour l'essor du tourisme au Groenland. L'activité est encore à ses balbutiements face à des destinations majeures positionnées sur le même produit touristique telles que l'Alaska, l'Islande ou la Patagonie, etc. Témoin plus anecdotique que scientifique, la date de réactualisation des guides Lonely Planet atteste du succès des ouvrages et conséquemment de la destination. Les derniers ouvrages sur la Patagonie, l'Islande et l'Alaska datent respectivement de 2009, 2010 et 2012. À l'inverse, le guide sur le Groenland n'a été l'objet d'aucune réactualisation depuis 2005 et les rares informations que Lonely Planet diffuse sont uniquement accessibles sur internet, témoignant du succès restreint de la destination.

\section{BIBLIOGRAPHIE}

ARNOULD P. et GLON É. (2006), “Wilderness, usages et perceptions de la nature en Amérique du Nord”, Annales de géographie, 649, pp. 227-238.

BAIDER F. (2003), “Construction de la mémoire, formulation de l'identité Chypre et les guides touristiques", Travaux de la Maison de l'Orient méditerranéen, 37, pp. 161- 173. 
BONIN S. (2001), "Paysages et représentations dans les guides touristiques. La Loire dans la collection des Guides-Joanne, Guides Bleus (1856 à nos jours)”, L'Espace géographique, 30, pp. 111-126.

CHEVALIER M. (1975), “Guides touristiques normands et idéologie touristique”, Norois, 88, 1, pp. 664- 667.

COHEN E. (1972), “Toward a sociology of international tourism”, Social research, 39, 1, pp. 164-182.

CRÉQUY A. (2010), “Le tourisme polaire. De la nature à la culture”, L'autre voie, 6.

ELSRUD T. (2001), "Risk creation in traveling backpacker adventure narration”, Annals of tourism research, 28, 3, p. 597-617.

GRAWITZ M. (1996), Méthodes des sciences sociales, Paris, Dalloz, 920 p.

GRENIER A. (2008), “Le tourisme de croisière”, Téoros, 27, 2, pp. 36-48.

GUILLET F. (2003), "Entre stratégie sociale et quête érudite : les notables normands et la fabrication de la Normandie au XIXe siècle”, Le Mouvement Social, 203, pp. 89- 111.

LARSEN S., ØGAARD T. et BRUN W. (2011), "Backpackers and mainstreamers Realities and myths", Annals of tourism research, 38, 2, pp. 690-707.

LEROUX S. (2007), "Les temporalités des touristes itinérants dans le sud marocain ou la quête de liberté”, Espace Population Sociétés, 2-3, pp. 273-284.

LÉVI-STRAUSS C. (1961), Race et histoire, Éditions Gonthier, 127 p.

LOKER-MURPHY L. et PEARCE, P. L. (1995), "Young budget travelers : backpackers in Australia”, Annals of tourism research, 22, 4, pp. 819-843.

MAOZ D. (2007), “Backpackers' motivations The role of culture and nationality”, Annals of tourism research, 34, 1, pp. 122-140.

MILL J. S. (2003), La nature, Paris, La découverte, 165 p.

NOY C. (2004), “This trip really changed me. Backpackers' narratives of self-change”, Annals of Tourism Research, 31, 1, pp. 78-102.

O'CARROLL E. et ELLIOTT M. (2005), Greenland and the Arctic, Hawthorn, Lonely Planet.

OLLIVIER G., GENIAUX G., NAPOLÉONE C., PAOLI C. (2012), "Mesurer la notoriété touristique communale : contribution méthodologique à l'aide de l'analyse textuelle de guides touristiques", Cybergeo : European Journal of Geography, http://cybergeo.revues.org/25187.

PEARCE P. L. et FOSTER F. (2007), “A 'University of Travel' : Backpacker learning”, Tourism management, 28, pp. 1285-1298.

SPERBER D. (1989), “L'étude anthropologique des représentation : problèmes et perspectives”, in JODELET D. (sous la direction), Les représentations sociales, Paris, PUF, pp. 133 à 148.

SWANEY D. (1991), Iceland, Greenland and the Faroe Islands, Hawthorn, Lonely Planet.

SWANEY D. (1994), Iceland, Greenland and the Faroe Islands, Hawthorn, Lonely Planet.

SWANEY D. (1999), The Arctic, Hawthorn, Lonely Planet.

SWANEY D. et CORNWALLIS G. (1997), Iceland, Greenland and the Faroe Islands, Hawthorn, Lonely Planet.

SWANEY D. et CORNWALLIS G. (2001), Iceland, Greenland and the Faroe Islands, Hawthorn, Lonely Planet. 


\section{NOTES}

1. Pluriel de inuk, ne prend pas la marque du pluriel.

2. Quantitativement il est impossible de déterminer l'ampleur de l'augmentation en raison de l'absence de séries statistiques pertinentes.

3. Terrain mené dans le cadre d'une thèse de géographie à l'université de Poitiers.

\section{RÉSUMÉS}

Cette étude livre une analyse diachronique des différents guides Lonely planet publiés sur le Groenland entre 1991 et 2005. Dans ces ouvrages les auteurs manifestent leur volonté de créer un "entre soi" propre au lectorat principal, les backpackers. La création de cette identité collective se fait par la critique des autres touristes et dans la manière singulière de découvrir la "wilderness" et la culture, principales raisons du succès touristique de l'île. Toutefois, cette identité collective semble remise en question par la confrontation du contenu des guides avec des travaux antérieurs et avec la réalité du terrain.

This article uses Lonely planet books published about Greenland between 1991 and 2005 to do a diachronic study. The central theme of these books is the will of their authors to highlight closeknit communities specific of main readership, backpackers. Criticize other tourists should be a way to create a collective identity and a singular way to discover wilderness and culture, the most important attractions of the island. Nevertheless this collective identity can be questioned in comparison to previous study and fieldwork.

\section{INDEX}

Keywords : tourism, backpackers, picturing, wilderness, Greenland, Lonely Planet Mots-clés : tourisme, backpackers, représentation, “entre soi”, Groenland, Lonely Planet

\section{AUTEUR}

\section{ANTOINE DELMAS}

Doctorant au laboratoire RURALITÉS, Université de Poitiers (France), Bureau 1.09, MSHS Bâtiment A5, 5 rue Théodore Lefebvre, 86000 Poitiers, Tél : 33 (0)5 494546 85, antoine.delmas@univ-poitiers.fr 